TRANSACTIONS OF THE

AMERICAN MATHEMATICAL SOCIETY

Volume 350, Number 5, May 1998, Pages 1895-1911

S 0002-9947(98)02150-3

\title{
A CONVEXITY THEOREM IN THE SCATTERING THEORY FOR THE DIRAC OPERATOR
}

\author{
K. L. VANINSKY
}

\begin{abstract}
The Dirac operator enters into zero curvature representation for the cubic nonlinear Schrödinger equation. We introduce and study a conformal map from the upper half-plane of the spectral parameter of the Dirac operator into itself. The action variables turn out to be limiting boundary values of the imaginary part of this map. We describe the image of the momentum map (convexity theorem) in the simplest case of a potential from the Schwartz class. We apply this description to the invariant manifolds for the nonlinear Schrödinger equation.
\end{abstract}

\section{INTRODUCTION}

The nonlinear Schrödinger (NLS) equation with repulsive potential ${ }^{1}$

$$
i \psi^{\bullet}=-\psi^{\prime \prime}+2|\psi|^{2} \psi
$$

has been solved for square integrable initial data on the circle and for a sumable potential on the entire line, see e.g. [MCV], [FT]. It is an important problem from statistical mechanics $[\mathrm{MCV}]$ to prove complete integrability for an extensive class of initial data which accommodate some translation-invariant Gibbs distribution on the entire line.

The integration of the NLS equation on the entire line is based on the scattering theory for the Dirac operator - the simplest $2 \times 2$ canonical system. In this paper we introduce a special conformal map (CM) from the upper-half plane of the spectral parameter into itself. The action variables, known before for the rapidly decreasing case in terms of so-called scattering coefficients, are defined now as a boundary value of the imaginary part of this map. The limiting boundary values are not a problem: they are known always to exist for a positive harmonic function [KO]. What we propose, in fact, is a continuum analog of the "Marchenko-Ostrovskii" conformal map $[\mathrm{MO}]$.

It is known that the Dirac operator is self-adjoint and that the Weyl-Titchmarsh functions are unique under the sole condition of continuity of the potential. We establish a relation between the boundary values of the CM with the Weyl-Titchmarsh functions, with the hope that, suitably interpreted, this relation defines a CM for a sufficiently broad class of potentials.

Received by the editors November 9, 1995 and, in revised form, June 21, 1996.

1991 Mathematics Subject Classification. Primary 34L05, 34L25, 34L40.

Key words and phrases. Scattering theory, convexity theorem, nonlinear Schrödinger.

The author would like to thank the Institut des Hautes Études Scientifiques, where the paper was completed, for hospitality. The work is partially supported by NSF grant DMS-9501002

1 - denotes a derivative in the time variable, ${ }^{\prime}$ in the $x$ variable. 
Construction of actions for the NLS equation with initial data from a particular function class reduces to the description of all possible boundary values of the CM, i.e. to the image of the momentum map. In this paper, we prove the simplest theorem of that type: for the Schwartz class $S\left(R^{1}\right)$ of rapidly decreasing infinitely smooth complex functions, the image of the momentum map is a convex cone of real non-negative functions likewise from $S\left(R^{1}\right)$. This is an infinite-dimensional generalization of the Atiyah-Guillemin-Sternberg convexity theorem [AT], [GS]. As an application of our result we solve the following problem.

Problem. What values of conventional local integrals of motion $H_{1}, H_{2}, \cdots, H_{N}$, $N<\infty$, where

$$
\begin{aligned}
& 2 H_{1}=\int|\psi|^{2} d x, \\
& 4 H_{2}=-i \int \bar{\psi} \psi^{\prime} d x, \\
& 8 H_{3}=\int\left|\psi^{\prime}\right|^{2}+|\psi|^{4} d x \quad \text { etc. }
\end{aligned}
$$

correspond to some invariant manifold in $S\left(R^{1}\right)$ ? What are all the invariant manifolds in $S\left(R^{1}\right)$ with given values of $H_{1}, H_{2}, \cdots, H_{N}$ ?

The paper is organized as follows. In sections 2 and 3 we present standard facts concerning Hamiltonian formalism and scattering theory needed in our considerations. The conformal map itself is introduced in section 4. It turns out that conventional integrals of NLS are the moments of the positive measure defined by the imaginary part of the CM. Section 5 establishes a relation between the boundary values of the CM, Weyl-Titchmarsh functions, and the spectral matrix. Section 6 contains necessary information about the periodic problem for the NLS equation. The relation between the CM and "Marchenko-Ostrovskii picture" is presented in section 7 . We also consider the whole structure in the linear limit. An interpretation of the results from the point of view of scattering theory is given in section 8 . In the two sections 9 and 10, we show that the actions are canonically paired to the angles, which are given in terms of the phase of the reflection coefficient. This demonstrates an action of the torus with continuum many generators, a standard requirement in convexity theorems. We exploit this structure to give an effective solution of the moment problem. The convexity theorem is presented in section 11. A conjecture about the image of the momentum map for potentials from the Sobolev class $H^{s}, s \geq 0$, is also stated there. The last section, 12, contains the solution of the problem.

The author is grateful to P. Deift, L. Faybusovich, H. McKean, V. Peller and N. Young for discussions.

\section{HAMiltonian FORMALiSM AND ZERO CURVATURE REPRESENTATION ${ }^{2}$}

We denote by $S\left(R^{1}\right)$ the Schwartz space of complex rapidly decreasing infinitely differentiable functions on the line such that

$$
\sup _{x}\left|\left(1+x^{2}\right)^{n} f^{(m)}(x)\right|<\infty \text { for all } n, m=0,1, \cdots .
$$

${ }^{2}$ The commutator representation for the NLS equation was found by [ZS]; see also [AKNS] for a more systematic approach. 
On the phase space $M \equiv S\left(R^{1}\right) \times S\left(R^{1}\right)$, which is a product of two copies of the Schwartz space, we introduce a Poisson bracket

$$
\{A, B\}=i \int_{R^{1}} \frac{\partial A}{\partial \psi} \frac{\partial B}{\partial \bar{\psi}}-\frac{\partial A}{\partial \bar{\psi}} \frac{\partial B}{\partial \psi} d x .
$$

The bracket is well-defined for any functionals $A, B$ with "nice" gradients. The original NLS equation

$$
i \psi^{\bullet}=-\psi^{\prime \prime}+2|\psi|^{2} \psi
$$

can be written in the form

$$
\psi^{\bullet}=\left\{8 H_{3}, \psi\right\} \quad \text { with } \quad 8 H_{3}=\int_{R^{1}}\left|\psi^{\prime}\right|^{2}+|\psi|^{4} d x=\text { energy } .
$$

There are two other classical integrals: $2 H_{1}=\int|\psi|^{2} d x=$ number of particles and $4 H_{2}=-i \int \bar{\psi} \psi^{\prime} d x=$ momentum. $H_{1}, H_{2}$ and $H_{3}$ are the first three Hamiltonians of an infinite hierarchy of commuting flows. NLS is a consistency condition for the zero-curvature representation

$$
\frac{\partial U}{\partial t}-\frac{\partial V_{3}}{\partial x}+[U, V]=0
$$

where $U=U_{0}+\lambda U_{1}$ with

$$
U_{0}=\left(\begin{array}{cc}
0 & \bar{\psi} \\
\psi & 0
\end{array}\right), \quad U_{1}=-i \sigma_{3},
$$

and $V_{3}=V_{0}+\lambda V_{1}+\lambda^{2} V_{2}$ with

$$
V_{0}=i\left(\begin{array}{cc}
|\psi|^{2} & -\bar{\psi}^{\prime} \\
\psi^{\prime} & -|\psi|^{2}
\end{array}\right), \quad V_{1}=-2 U_{2}, \quad V_{2}=-4 U_{1} .
$$

Here and below $\sigma$ denotes the Pauli matrices

$$
\sigma_{1}=\left(\begin{array}{cc}
0 & 1 \\
1 & 0
\end{array}\right), \quad \sigma_{2}=\left(\begin{array}{cc}
0 & -i \\
i & 0
\end{array}\right), \quad \sigma_{3}=\left(\begin{array}{cc}
1 & 0 \\
0 & -1
\end{array}\right) .
$$

\section{THE REDUCED MONODROMY MATRIX}

The results of this section are basically well known. They are included here to make the paper self-contained. ${ }^{3}$ The standard monodromy matrix $M(x, y, \lambda)$ is the $2 \times 2$ solution of

$$
M^{\prime}(x, y, \lambda)=U(x, \lambda) M(x, y, \lambda),\left.\quad M(x, y, \lambda)\right|_{x=y}=I,
$$

where $I$ is the identity matrix. The reduced monodromy matrix $T(x, y, \lambda)$ is defined as

$$
T(x, y, \lambda) \equiv E^{-1}(\lambda x) M(x, y, \lambda) E^{-1}(-\lambda y),
$$

where $E(\lambda x)=\exp \left(-i \lambda x \sigma_{3}\right)$ is a solution of the free equation $(\psi \equiv 0)$. The matrix $T$ solves

$$
T^{\prime}(x, y, \lambda)=U_{0}(x) E(2 \lambda x) T(x, y, \lambda),\left.\quad T(x, y, \lambda)\right|_{x=y}=I .
$$

It can be expressed as

$$
T(x, y, \lambda)=\stackrel{\Re}{\exp } \int_{y}^{x} U_{0}(\xi) E(2 \lambda \xi) d \xi .
$$

\footnotetext{
${ }^{3}$ A study of the scattering theory for canonical systems goes back to $[\mathrm{K}],[\mathrm{KMA}],[\mathrm{MA}]$. For the latest results see $[\mathrm{BDZ}]$.
} 
Note that both $M$ and $T$ belong to $S L(2, \mathbb{C})$. The symmetry of the matrix $U$ : $\sigma_{1} U_{0}(x) \sigma_{1}=\overline{U_{0}(x)}$ is inherited by $T: \sigma_{1} T(x, y, \bar{\lambda}) \sigma_{1}=\overline{T(x, y, \lambda)}$.

For $\psi \in S\left(R^{1}\right)$ and $\lambda$ real, the existence of the limit

$$
\lim T(x, y, \lambda)=T(\lambda)=\left(\begin{array}{ll}
a(\lambda) & b^{\star}(\lambda) \\
b(\lambda) & a^{\star}(\lambda)
\end{array}\right), \quad \text { for } \quad y \rightarrow-\infty \quad \text { and } \quad x \rightarrow+\infty
$$

follows easily from the inequality

$$
\left|\stackrel{\curvearrowleft}{\exp } \int_{y}^{x} U_{0}(\xi) E(2 \lambda \xi) d \xi-I\right| \leq \exp \int_{y}^{x}\left|U_{0}(\xi)\right| d \xi-1,
$$

where $|\bullet|$ is any multiplicative matrix norm. Due to the symmetry of $T(x, y, \lambda)$, $T(\lambda)$ has the form

$$
\left(\begin{array}{ll}
a(\lambda) & \bar{b}(\lambda) \\
b(\lambda) & \bar{a}(\lambda)
\end{array}\right) \quad \text { with } \quad|a(\lambda)|^{2}-|b(\lambda)|^{2}=1
$$

The important fact needed bellow is that $b(\lambda) \in S\left(R^{1}\right)$. This implies the following property of $a(\lambda)$

$i$. For $\lambda$ real, $|a(\lambda)| \geq 1$ and $|a(\lambda)|^{2}-1 \in S\left(R^{1}\right)$.

To establish properties of $T(\lambda)$ for complex $\lambda$, we introduce the Jost solutions $M_{ \pm}(x, \lambda)$ :

$$
M_{ \pm}^{\prime}(x, \lambda)=U(x, \lambda) M_{ \pm}(x, \lambda), \quad M_{ \pm}(x)=E(\lambda x)+o(1) \quad \text { as } \quad x \rightarrow \pm \infty .
$$

They can be expressed through the solution of the free equation by means of transformation operators:

$$
\begin{aligned}
& M_{+}(x, \lambda)=E(x \lambda)+\int_{x}^{+\infty} \Gamma_{+}(x, \xi) E(\lambda \xi) d \xi \\
& M_{-}(x, \lambda)=E(x \lambda)+\int_{-\infty}^{x} \Gamma_{-}(x, \xi) E(\lambda \xi) d \xi .
\end{aligned}
$$

Integral equations and estimates for the kernels $\Gamma_{ \pm}$are derived following $[\mathrm{M}$, section 3.1].

The kernels $\Gamma_{ \pm}$are unique and infinitely smooth in both variables. They have a symmetry $\bar{\Gamma}_{ \pm}=\sigma_{1} \Gamma_{ \pm} \sigma_{1}$ and admit unique decompositions $\Gamma_{ \pm}=C_{ \pm}+A_{ \pm}$, where $C_{ \pm}$, resp. $A_{ \pm}$commute, resp. anti-commute with $\sigma_{1}$. The components $C_{-}, A_{-}$of the kernel $\Gamma_{-}$satisfy

$$
\begin{aligned}
& C_{-}(x, t)=\int_{-\infty}^{x} U_{0}(\xi) A_{-}(\xi, t+\xi-x) d \xi \\
& A_{-}(x, t)=\frac{1}{2} U_{0}\left(\frac{t+x}{2}\right)+\int_{\frac{t+x}{2}}^{x} U_{0}(\xi) C_{-}(\xi, t+x-\xi) d \xi,
\end{aligned}
$$

whence

$$
A_{-}(x, x)=\frac{1}{2} U_{0}(x), \quad C_{-}(x, x)=\frac{1}{2} \int_{-\infty}^{x} U_{0}^{2}(\xi) d \xi .
$$


The components $C_{+}, A_{+}$of the kernel $\Gamma_{+}$also satisfy

$$
\begin{aligned}
& C_{+}(x, t)=-\int_{x}^{+\infty} U_{0}(\xi) A_{+}(\xi, t+\xi-x) d \xi \\
& A_{+}(x, t)=-\frac{1}{2} U_{0}\left(\frac{t+x}{2}\right)-\int_{x}^{\frac{t+x}{2}} U_{0}(\xi) C_{+}(\xi, t+x-\xi) d \xi,
\end{aligned}
$$

whence

$$
A_{+}(x, x)=-\frac{1}{2} U_{0}(x), \quad C_{+}(x, x)=\frac{1}{2} \int_{x}^{\infty} U_{0}^{2}(\xi) d \xi .
$$

The monodromy matrix $M(x, y, \lambda)$ can be written in the form

$$
M(x, y)=M_{+}(x) M_{+}^{-1}(y)=M_{-}(x) M_{-}^{-1}(y) .
$$

Therefore

$$
M_{+}^{-1}(x) M(x, y) M_{-}(y)=M_{+}^{-1}(y) M_{-}(y)=M_{+}^{-1}(x) M_{-}(x) .
$$

The expression at the right does not depend on $x$ or $y$ and is equal to $T(\lambda)$, as one can see by passing to the limit as $x \rightarrow+\infty, y \rightarrow-\infty$. Introducing the notation $M_{ \pm}=\left(M_{ \pm}^{1}, M_{ \pm}^{2}\right)$ for the columns, we get $a(\lambda)=\operatorname{det}\left(M_{-}^{1}, M_{+}^{2}\right)$. Now the following properties of $a(\lambda)$ follow easily from the integral representation of the Jost solutions:

ii. $a(\lambda)$ is analytic in the upper half-plane and infinitely smooth up to the boundary;

iii. $a(\lambda)=1+o(1)$ as $|\lambda| \longrightarrow \infty$, and $a(\lambda)$ is root-free.

\section{The CONFORMal MAP $p_{\infty}(\lambda)$}

Let $p_{\infty}(\lambda)$ be such that $a(\lambda)=\exp \left(-i p_{\infty}(\lambda)\right)$ for $\lambda$ in the upper half-plane. From the properties of $a(\lambda)$ presented above follow:

$i$. $p_{\infty}(\lambda)$ is analytic in the upper half-plane and infinitely smooth up to the boundary.

ii. $\Im p_{\infty}(\lambda) \geq 0$ for $\Im \lambda \geq 0$.

This means simply that $p_{\infty}(\lambda)$ defines a conformal map from the upper half-plane of the spectral parameter into itself. Moreover,

iii. $p_{\infty}(\lambda)=o(1)$ for $|\lambda| \rightarrow \infty$; for real $\lambda$, the density of the measure $d \mu_{\infty}(\lambda)=$ $\Im p_{\infty}(\lambda) d \lambda$ belongs to $S\left(R^{1}\right)$ and $d \mu_{\infty}(\lambda)$ has moments of any order.

This is due to the fact that $|a(\lambda)|^{2}-1 \in S\left(R^{1}\right)$. The function $p_{\infty}(\lambda)$ can be written in the form

$$
p_{\infty}(\lambda)=\frac{1}{\pi} \int \frac{d \mu_{\infty}(t)}{t-\lambda},
$$

see $[\mathrm{A}]$. Expanding the denominator in inverse powers of $\lambda$, we obtain

$$
p_{\infty}(\lambda)=-\sum_{k=0}^{\infty} \frac{1}{\lambda^{k+1}} \frac{1}{\pi} \int_{-\infty}^{+\infty} t^{k} d \mu_{\infty}(t)=-\frac{H_{1}}{\lambda}-\frac{H_{2}}{\lambda^{2}}-\frac{H_{3}}{\lambda^{3}}-\ldots,
$$

where $H_{1}, H_{2}$ and $H_{3}$ are the integrals introduced above. The expansion has an asymptotic character for $\lambda$ when $\delta \leq \arg \lambda \leq \pi-\delta$. An identification of the 
coefficients of the asymptotic expansion employs a recurrence relation for $H$ 's. As in $[\mathrm{MCV}]$, a straightforward computation produces

$$
\begin{aligned}
& D \frac{1}{2 \bar{\psi}}\left[2 i \lambda \frac{\partial T}{\partial \psi(x)}+D \frac{\partial T}{\partial \psi(x)}\right]=\psi \frac{\partial T}{\partial \psi}-\bar{\psi} \frac{\partial T}{\partial \bar{\psi}}, \\
& D \frac{1}{2 \psi}\left[2 i \lambda \frac{\partial T}{\partial \bar{\psi}(x)}-D \frac{\partial T}{\partial \bar{\psi}(x)}\right]=\psi \frac{\partial T}{\partial \psi}-\bar{\psi} \frac{\partial T}{\partial \bar{\psi}} .
\end{aligned}
$$

Here we have used only the formulas for derivatives:

$$
\begin{aligned}
\frac{\partial T}{\partial \psi(x)} & =T(+\infty, x)\left(\begin{array}{ll}
0 & 0 \\
1 & 0
\end{array}\right) E(2 \lambda x) T(x,-\infty), \\
\frac{\partial T}{\partial \bar{\psi}(x)} & =T(+\infty, x)\left(\begin{array}{ll}
0 & 1 \\
0 & 0
\end{array}\right) E(2 \lambda x) T(x,-\infty) .
\end{aligned}
$$

Now we can put the formulas into the desired form of a recurrence relation:

$$
J \nabla H_{n+1}=K \nabla H_{n}, \quad n=1,2, \ldots,
$$

with $^{4}$

$$
K=\left(\begin{array}{cc}
-i \psi D^{-1} \psi & -i / 2 D+i \psi D^{-1} \bar{\psi} \\
-i / 2 D+i \bar{\psi} D^{-1} \psi & -i \bar{\psi} D^{-1} \bar{\psi}
\end{array}\right) \quad \text { and } \quad J=i \sigma_{2} .
$$

This allows us to reconstruct the $H$ 's starting from $2 H_{1}=\int|\psi|^{2}$. The same recurrence relation implies an involutive character of $H$ 's; see [MC1].

\section{The Relation Between $p_{\infty}(\lambda)$, Weyl-Titchmarsh Functions} AND THE SPECTRAL MATRIX

By definition, the Weyl solutions $h_{ \pm}=\left(\begin{array}{c}h_{ \pm}^{1} \\ h_{ \pm}^{2}\end{array}\right)$ of $h^{\prime}=U h$ belong, resp., to $L^{2}\left(R_{ \pm}^{1}\right)$ for $\lambda$ with strictly positive imaginary part; they are normalized by $h_{+}^{2}(x, \lambda)$ $=1$, resp., $h_{-}^{1}(x, \lambda)=1$ for $x=0$. They can be written in the form

$$
\begin{aligned}
& h_{+}(x, \lambda)=M^{1}(x, \lambda) m_{+}(\lambda)+M^{2}(x, \lambda), \\
& h_{-}(x, \lambda)=M^{1}(x, \lambda)+M^{2}(x, \lambda) m_{-}(\lambda),
\end{aligned}
$$

where $M(x, \lambda) \equiv M(x, 0, \lambda)$. For example, for the free equation, the Weyl-Titchmarsh functions $m_{ \pm}$are identically zero and

$$
\begin{aligned}
& h_{+}(x, \lambda)=\left(\begin{array}{c}
0 \\
e^{i \lambda x}
\end{array}\right) \in L^{2}\left(R_{+}^{1}\right), \\
& h_{-}(x, \lambda)=\left(\begin{array}{c}
e^{-i \lambda x} \\
0
\end{array}\right) \in L^{2}\left(R_{-}^{1}\right) .
\end{aligned}
$$

It is a well-known fact, see [LS], that under the sole assumption of the continuity of the potential $\psi$, the functions $m_{ \pm}$are unique.

Our first goal is to prove that

$$
e^{2 \Im p_{\infty}(\lambda)}=\frac{\left|1-m_{-} m_{+}\right|^{2}}{\left(1-\left|m_{+}\right|^{2}\right)\left(1-\left|m_{-}\right|^{2}\right)} \quad \text { for } \lambda \text { real. }
$$

This formula provides an alternative way to define the CM.

$$
{ }^{4} D^{-1}=\frac{1}{2}\left(\int_{-\infty}^{x}-\int_{x}^{\infty}\right) .
$$


Note that $M_{+}^{2}$, resp. $M_{-}^{1}$, are from $L^{2}\left(R_{+}^{1}\right)$, resp. $L^{2}\left(R_{-}^{1}\right)$, for $\lambda$ with strictly positive imaginary part. Therefore

$$
M_{-}^{1}=k_{-}(\lambda) h_{-}, \quad M_{+}^{2}=k_{+}(\lambda) h_{+},
$$

for some numbers $k_{ \pm}(\lambda)$. Take $\psi$ supported in $[-L,+L]$. Then

$$
M(0, L) M_{+}^{2}(L)=M_{+}^{2}(0)=k_{+} h_{-}(0) .
$$

In more detail,

$$
\left(\begin{array}{ll}
m_{11} & m_{12} \\
m_{21} & m_{22}
\end{array}\right)(0, L)\left(\begin{array}{c}
0 \\
e^{i \lambda L}
\end{array}\right)=\left(\begin{array}{c}
m_{+} \\
1
\end{array}\right) k_{+},
$$

or

$$
m_{22} e^{i \lambda L}=k_{+}, \quad m_{12} e^{i \lambda L}=m_{+} k_{+}
$$

Finally, for $\lambda$ real

$$
1=\left|m_{22}\right|^{2}-\left|m_{12}\right|^{2}=\left|k_{+}\right|^{2}\left(1-\left|m_{+}\right|^{2}\right) \quad \text { and } \quad\left|k_{+}\right|^{2}=\frac{1}{1-\left|m_{+}\right|^{2}}
$$

and likewise

$$
\left|k_{-}\right|^{2}=\frac{1}{1-\left|m_{-}\right|^{2}}
$$

Therefore, for such $\lambda$,

$$
\begin{aligned}
e^{2 \Im p_{\infty}(\lambda)} & =|a(\lambda)|^{2}=\left|\operatorname{det}\left(M_{-}^{1}, M_{+}^{2}\right)\right|^{2}=\left|k_{-} k_{+}\left(1-m_{-} m_{+}\right)\right|^{2} \\
& =\frac{\left|1-m_{-} m_{+}\right|^{2}}{\left(1-\left|m_{+}\right|^{2}\right)\left(1-\left|m_{-}\right|^{2}\right)}
\end{aligned}
$$

as required.

The first order system $F^{\prime}=U F$ introduced at the beginning can be expressed as an eigenvalue problem for the Dirac operator $\mathfrak{D}$ :

$$
\mathfrak{D} f=\left[\left(\begin{array}{ll}
1 & 0 \\
0 & 1
\end{array}\right) i D+\left(\begin{array}{cc}
0 & -i \bar{\psi} \\
i \psi & 0
\end{array}\right)\right] f=\lambda f .
$$

The Spectral Theorem. For the operator $\mathfrak{D}$ with a continuous potential there exists a unique $2 \times 2$ Hermitian spectral matrix $\left\|\rho_{i j}(\lambda)\right\| ;\left\|\rho_{i j}\left(\lambda^{\prime}\right)-\rho_{i j}\left(\lambda^{\prime \prime}\right)\right\|$ is positive definite for any real $\lambda^{\prime}>\lambda^{\prime \prime}$; the entries $\rho_{i j}(\lambda)$ are functions continuous from the right and

$$
\int \frac{\left|d \rho_{i j}(\lambda)\right|}{1+\lambda^{2}}<\infty
$$

For any vector-function $f \in L^{2}\left(R^{1}\right)$, the sequence

$$
F_{n}^{i}(\lambda)=\int_{-n}^{+n} f^{T}(x) \bar{M}^{i}(x, \lambda) d x, \quad i=1,2,
$$

converges to some $F_{i}(\lambda)$ in $L^{2}\left(R^{1}, \rho_{i j}\right)$ :

$$
\int \sum_{i j}\left(F_{n}^{i}-F^{i}\right) \overline{\left(F_{n}^{j}-F^{j}\right)} d \rho_{i j} \rightarrow \infty, \quad \text { as } \quad n \rightarrow \infty .
$$

Moreover,

$$
\int|f|^{2} d x=\int \sum_{i j} F^{i}(\lambda) \overline{F^{j}(\lambda)} d \rho_{i j}(\lambda) .
$$


The function $\rho_{i i}, i=1,2$, is the boundary value of the expression

$$
\frac{1}{2 \pi} \frac{1-\left|m_{-}\right|^{2}\left|m_{+}\right|^{2}}{\left|1-m_{-} m_{+}\right|^{2}}
$$

and $\rho_{12}=\overline{\rho_{21}}$ is the boundary value of the expression

$$
\frac{1}{2 \pi} \frac{\overline{m_{+}}\left(1-\left|m_{-}\right|^{2}\right)-m_{-}\left(1-\left|m_{+}\right|^{2}\right)}{\left|1-m_{-} m_{+}\right|^{2}} .
$$

The proof is by standard arguments, see for example [CL]. By direct computation,

$$
\operatorname{det}\left\|\rho_{i j}\right\|=\frac{1}{4 \pi^{2}} \frac{\left(1-\left|m_{+}\right|^{2}\right)\left(1-\left|m_{-}\right|^{2}\right)}{\left|1-m_{-} m_{+}\right|^{2}},
$$

which implies $\operatorname{det}\left\|\rho_{i j}(\lambda)\right\|=e^{-2 \Im p_{\infty}(\lambda)} / 4 \pi^{2}$ for $\lambda$ real. ${ }^{5}$

One can think about a spectral matrix as a smooth functional on the phase space. Indeed, gradients of the functions $m_{ \pm}$can be computed, e.g.

$$
\begin{aligned}
& \frac{\partial m_{+}(\lambda)}{\partial \psi(y)}=\left[h_{+}^{1}(y, \lambda)\right]^{2}, \\
& \frac{\partial m_{+}(\lambda)}{\partial \bar{\psi}(y)}=-\left[h_{+}^{2}(y, \lambda)\right]^{2} .
\end{aligned}
$$

We split the proof of the first formula into 5 steps.

Step 1. By direct computation

$$
\begin{aligned}
& \frac{\partial M(x, 0)}{\partial \psi(y)}=M(x, y)\left(\begin{array}{ll}
0 & 0 \\
1 & 0
\end{array}\right) M(y, 0), \\
& \frac{\partial M(x, 0)}{\partial \bar{\psi}(y)}=M(x, y)\left(\begin{array}{ll}
0 & 1 \\
0 & 0
\end{array}\right) M(y, 0),
\end{aligned}
$$

where $0 \leq y \leq x$.

Step 2. Let $f, g$ be two vector solutions of $\mathfrak{D} f=\lambda f$; then $\operatorname{det}[f(x), g(x)]$ does not depend on $x$. The proof is trivial.

Step 3. The Weyl solution $h_{+}$, normalized by $\left.h_{+}^{2}(x, y, \lambda)\right|_{x=y}=1$, can be expressed as

$$
h_{+}(x, y, \lambda)=M^{1}(x, y, \lambda) m_{+}(\lambda, y)+M^{2}(x, y, \lambda),
$$

where $m_{+}(\lambda, y)$ is the corresponding Weyl-Titchmarsh function. In particular, $h_{+}(x, \lambda)=h_{+}(x, 0, \lambda)$. The fact needed below is that

$$
m_{+}(\lambda, y)=\frac{h_{+}^{1}(y, 0, \lambda)}{h_{+}^{2}(y, 0, \lambda)} .
$$

To prove this, note that the identity $M(x, 0)=M(x, y) M(y, 0)$ implies

$$
\begin{aligned}
& M^{1}(x, 0)=m_{11}(y, 0) M^{1}(x, y)+m_{21}(y, 0) M^{2}(x, y), \\
& M^{2}(x, 0)=m_{12}(y, 0) M^{1}(x, y)+m_{22}(y, 0) M^{2}(x, y) .
\end{aligned}
$$

\footnotetext{
${ }^{5}$ H.P. McKean in [MC2] proved a similar formula.
} 
Therefore, for $0 \leq y \leq x$,

$$
\begin{aligned}
h_{+}(x, 0, \lambda)= & M^{1}(x, 0) m_{+}(\lambda, 0)+M^{2}(x, 0) \\
= & {\left[m_{11}(y, 0) M^{1}(x, y)+m_{21}(y, 0) M^{2}(x, y)\right] m_{+}(\lambda, 0) } \\
& \quad+\left[m_{12}(y, 0) M^{1}(x, y)+m_{22}(y, 0) M^{2}(x, y)\right] \\
= & {\left[m_{21}(y, 0) m_{+}(\lambda, 0)+m_{22}(y, 0)\right] } \\
& \quad \times\left[M^{1}(x, y) \frac{m_{11}(y, 0) m_{+}(\lambda, 0)+m_{12}(y, 0)}{m_{21}(y, 0) m_{+}(\lambda, 0)+m_{22}(y, 0)}+M^{2}(x, y)\right] .
\end{aligned}
$$

The result follows.

Step 4. The purpose of this step is to prove the formula

$$
\frac{\partial m_{+}(\lambda, 0)}{\partial \psi(y)}=-m_{+}(\lambda, 0) m_{+}(\lambda, y)\left[\frac{m_{12}(y, 0)}{A}+\frac{m_{11}(y, 0)}{B}\right]
$$

where

$$
\begin{aligned}
& A=m_{12}(y, 0)-m_{+}(\lambda, y) m_{22}(y, 0), \\
& B=-m_{11}(y, 0)+m_{+}(\lambda, y) m_{21}(y, 0) .
\end{aligned}
$$

Consider the eigenvalue problem $\mathfrak{D} f=\lambda f$ with boundary conditions $f^{2}(y, \lambda)$ $=1, f^{1}(b, \lambda)=f^{2}(b, \lambda)$, where $0 \leq y<b<+\infty$. The solution can be written in the form

$$
f(x, \lambda)=M^{1}(x, y, \lambda) m_{+}(\lambda, y, b)+M^{2}(x, y, \lambda) .
$$

Due to the selfadjointness of the spectral problem, the limit

$$
m_{+}(\lambda, y)=\lim _{b \rightarrow+\infty} m_{+}(\lambda, y, b)
$$

exists and does not depend on the boundary condition at the right end of the interval. From the definition,

$$
m_{+}(\lambda, y, b)=\frac{m_{22}-m_{12}}{m_{11}-m_{21}}(b, y, \lambda)
$$

Therefore,

$$
\begin{aligned}
\nabla m_{+}(\lambda, 0, b)= & \frac{m_{22}-m_{12}}{m_{11}-m_{21}} \frac{\nabla m_{22}-\nabla m_{12}}{m_{22}-m_{12}}(b, 0, \lambda) \\
& -\frac{m_{22}-m_{12}}{m_{11}-m_{21}} \frac{\nabla m_{11}-\nabla m_{21}}{m_{11}-m_{21}}(b, 0, \lambda) .
\end{aligned}
$$

By Step 1,

$$
\begin{aligned}
\frac{\partial m_{+}(\lambda, 0, b)}{\partial \psi(y)}= & m_{+}(\lambda, 0, b) \frac{\left(m_{22}-m_{12}\right)(b, y) m_{12}(y, 0)}{\left(m_{22}-m_{12}\right)(b, 0)} \\
& -m_{+}(\lambda, 0, b) \frac{\left(m_{12}-m_{22}\right)(b, y) m_{11}(y, 0)}{\left(m_{11}-m_{21}\right)(b, 0)} \\
= & m_{+}(\lambda, 0, b)\left(m_{22}-m_{12}\right)(b, y) \\
& \times\left[\frac{m_{12}(y, 0)}{\left(m_{22}-m_{12}\right)(b, 0)}+\frac{m_{11}(y, 0)}{\left(m_{11}-m_{21}\right)(b, 0)}\right] .
\end{aligned}
$$


Using the identity $M(b, 0)=M(b, y) M(y, 0)$, and simple algebra, one finds the quantity

$$
\begin{aligned}
& -m_{+}(\lambda, 0, b) m_{+}(\lambda, y, b) \\
& \quad \times\left[\frac{m_{12}(y, 0)}{m_{12}(y, 0)-m_{+}(\lambda, y, b) m_{22}(y, 0)}+\frac{m_{11}(y, 0)}{-m_{11}(y, 0)+m_{+}(\lambda, y, b) m_{21}(y, 0)}\right] .
\end{aligned}
$$

Now pass to the limit as $b \rightarrow \infty$.

Step 5. By Step 3,

$$
m_{+}(\lambda, y)\left[m_{21}(y, 0) m_{+}(\lambda, 0)+m_{22}(y, 0)\right]=m_{11}(y, 0) m_{+}(\lambda, 0)+m_{12}(y, 0) .
$$

After simple algebra,

$$
\frac{m_{+}(\lambda, 0)}{A}=\frac{1}{B}
$$

Therefore,

$$
\frac{\partial m_{+}(\lambda, 0)}{\partial \psi(y)}=-m_{+}(\lambda, y)\left[\frac{m_{+}(\lambda, 0) m_{11}(y, 0)+m_{12}(y, 0)}{-m_{11}(y, 0)+m_{21}(y, 0) m_{+}(\lambda, y)}\right] .
$$

By Step 3,

$$
\begin{aligned}
\frac{\partial m_{+}(\lambda, 0)}{\partial \psi(y)} & =\frac{h_{+}^{1}(y, \lambda)}{m_{11}(y, 0) \frac{h_{+}^{2}(y, 0)}{h_{+}^{1}(y, 0)}-m_{21}(y, 0)} \\
& =\frac{\left[h_{+}^{1}(y, \lambda)\right]^{2}}{m_{11}(y, 0) h_{+}^{2}(y, 0)-m_{21}(y, 0) h_{+}^{1}(y, 0)} .
\end{aligned}
$$

By Step 2, the denominator can be computed at $y=0$, where it is equal to 1 . We are done.

The proof of the second formula is the same.

\section{The NeCESSARY information ABOUt PERIOdiC PRoblem ${ }^{6}$}

In the next two sections we relate the $\mathrm{CM}$ defined by $p_{\infty}(\lambda)$ with the analogous object known in the periodic case. Consider the periodic problem for the NLS, i.e. $x$ is on the circle of perimeter $2 L$, and assume that the function $\psi$ is infinitely smooth. The periodic/anti-periodic spectrum of the auxiliary spectral problem is determined by

$$
\Delta_{L}(\lambda) \equiv \operatorname{trace} M(L,-L, \lambda) / 2 .
$$

Namely, $\lambda_{0}$ is a periodic, resp. anti-periodic, eigenvalue if it is a root of the equation $\Delta_{L}\left(\lambda_{0}\right)=+1$, resp. -1 . The function $\pi_{L}(\lambda)$ is defined by $\Delta_{L}(\lambda)=\cosh i \pi_{L}(\lambda)$; it has the properties

i. $\pi_{L}(\lambda)$ is single-valued on the $\lambda$-plane cut along open gaps $\left(\lambda \in R^{1}:\left|\Delta_{L}(\lambda)\right|\right.$ $>1)$

ii. $\bar{\pi}_{L}(\lambda)=\pi_{L}(\bar{\lambda})$

iii. $\Im \pi_{L}(\lambda) \geq 0$ for $\lambda$ in the upper half-plane.

\footnotetext{
${ }^{6}$ For more information see [MCV].
} 
The function $\pi_{L}(\lambda)$ determines a conformal map from the upper half-plane into a slit-domain. In fact, this CM was known to physicists already in 1929, see [B]. This map was used by $[\mathrm{MO}]$ in their description of all possible spectral sequences for the periodic Schrödinger operator.

The function $\pi_{L}(\lambda)$ can be written in the form

$$
\pi_{L}(\lambda)=2 \lambda L+p_{L}(\lambda)
$$

in which

i. $p_{L}(\lambda)$ is analytic and continuous up to the boundary;

ii. $\bar{p}_{L}(\lambda)=p_{L}(\bar{\lambda})$

iii. $\Im p_{L}(\lambda) \geq 0$ for $\lambda$ in the upper half-plane; the inequality is strict, except for two simple zeroes, one in each band.

Introducing a measure $d \mu_{L}(\lambda) \equiv \Im p_{L}(\lambda) d \lambda$, we can write $p_{L}(\lambda)$ in terms of the Cauchy integral:

$$
p_{L}(\lambda)=\frac{1}{\pi} \int \frac{d \mu_{L}(t)}{t-\lambda} .
$$

The function $p_{L}(\lambda)$ has an asymptotic expansion at infinity analogous to the asymptotic expansion of $p_{\infty}(\lambda)$ :

$$
p_{L}(\lambda)=\pi_{L}(\lambda)-2 \lambda L=-\sum_{k=0}^{\infty} \frac{1}{\lambda^{k+1}} \frac{1}{\pi} \int_{-\infty}^{+\infty} t^{k} d \mu_{L}(t)=-\frac{H_{1}}{\lambda}-\frac{H_{2}}{\lambda^{2}}-\frac{H_{3}}{\lambda^{3}}-\ldots
$$

\section{The infinite-volume limit. Deformation to the linear problem}

The scaling $\psi \rightarrow \sqrt{\varkappa} \psi, \varkappa>0$, transforms the original NLS equation into

$$
i \psi^{\bullet}=-\psi^{\prime \prime}+2 \varkappa|\psi|^{2} \psi .
$$

We denote by $p_{\infty}^{\varkappa}(\lambda), \psi_{L}^{\varkappa}(\lambda)$, etc., all the quantities introduced before to emphasize their dependence on the parameter $\varkappa$. The function $p_{\infty}^{\varkappa}(\lambda)$ turns out to be a continuum analog of $p_{L}^{\varkappa}(\lambda)$. Namely, for $\lambda$ with strictly positive imaginary part,

$$
p_{L}^{\varkappa}(\lambda) \longrightarrow p_{\infty}^{\varkappa}(\lambda) \quad \text { as } L \rightarrow \infty
$$

To prove this, fix a compactly supported potential. Then $T(\lambda)$ is an entire matrixfunction, as follows from the matrix exponential. Consider now the identity

$$
T(L,-L, \lambda)=E(\lambda L) T(\lambda) E(\lambda L)=\left(\begin{array}{cc}
a(\lambda) e^{-2 i \lambda L} & b^{\star} \\
b(\lambda) & a^{\star}(\lambda) e^{2 i \lambda L}
\end{array}\right) .
$$

Taking the trace of both sides, we get

$$
2 \Delta_{L}^{\varkappa}(\lambda)=e^{i\left(2 \lambda L+\psi_{L}^{\varkappa}(\lambda)\right)}+e^{-i\left(2 \lambda L+\psi_{L}^{\varkappa}(\lambda)\right)}=e^{-2 i \lambda L} a(\lambda)+a^{\star}(\lambda) e^{2 i \lambda L} .
$$

Therefore, the difference $e^{-i \psi_{L}^{\varkappa}(\lambda)}-e^{-i p_{L}^{\varkappa}(\lambda)}=-e^{4 i \lambda L}\left[e^{\psi_{L}^{\varkappa}(\lambda)}+a^{\star}(\lambda)\right]$ is negligible as $L \rightarrow \infty$. That gives us a second proof that $p_{\infty}(\lambda)$ has a positive imaginary part.

The deformation of the measure $d \mu^{\varkappa}$ in the infinite-volume or linear limit can best be expressed by a "commutative" diagram:

$$
\begin{aligned}
& d \mu_{L}^{\varkappa}(\lambda) \quad \stackrel{L \rightarrow \infty}{\longrightarrow} \quad d \mu_{\infty}^{\varkappa}(\lambda) \\
& \varkappa^{-1} d \mu_{L}^{\varkappa}(\lambda), \varkappa \downarrow 0 \downarrow \quad \downarrow \varkappa^{-1} d \mu_{\infty}^{\varkappa}(\lambda), \varkappa \downarrow 0 \\
& d \mu_{L}^{0}(\lambda)=\sum_{n} \delta\left(\lambda-\frac{\pi n}{L}\right)|\hat{\psi}(n)|^{2} d \lambda \stackrel{L \rightarrow \infty}{\longrightarrow} d \mu_{\infty}^{0}(\lambda)=\frac{1}{2 \pi}|\hat{\psi}(\lambda)|^{2} d \lambda,
\end{aligned}
$$


where

$$
\hat{\psi}(n)=\frac{1}{2 L} \int_{-L}^{+L} e^{-i x \pi n / L} \psi(x) d x, \quad \hat{\psi}(\lambda)=\int_{R^{1}} e^{-i x \lambda} \psi(x) d x .
$$

The convergence of measures has to be understood in the weak* sense.

\section{THE SCATTERING THEORY}

This section is a brief retreat into physics. It explains on physical grounds why $p_{\infty}(\lambda)$ has a positive imaginary part. Consider two solutions $f_{l}$ and $f_{r}$ with prescribed asymptotics as $x \rightarrow \pm \infty$ :

$$
\begin{aligned}
& x \rightarrow-\infty \quad x \rightarrow+\infty \\
& f_{l} \quad f_{\rightarrow}+B(\lambda) f_{\leftarrow} \quad A(\lambda) f_{\rightarrow} \\
& f_{r} \quad D(\lambda) f_{\leftarrow} \quad f_{\leftarrow+C}+C(\lambda) f_{\rightarrow}
\end{aligned}
$$

where

$$
f_{\leftarrow}=\left(\begin{array}{c}
e^{-i \lambda x} \\
0
\end{array}\right), \quad f_{\rightarrow}=\left(\begin{array}{c}
0 \\
e^{i \lambda x}
\end{array}\right)
$$

are the solutions of the free equation. The solutions $f_{l}$, resp. $f_{r}$, describe a particle which approaches a potential barrier from the left, resp. right. The coefficients $B$, resp. $C$, are called left, resp. right reflection coefficients, and $A=D$ is a transmission coefficient.

As in section 5, we can compute a scattering matrix $S(\lambda)$ :

$$
S(\lambda) \equiv\left(\begin{array}{ll}
A & B \\
C & D
\end{array}\right)=\left(\begin{array}{cc}
1 / a(\lambda) & -\bar{b}(\lambda) / a(\lambda) \\
b(\lambda) / a(\lambda) & 1 / a(\lambda)
\end{array}\right) .
$$

Compute the probability for the particle to go through or be reflected by the barrier:

Prob $\{$ particle goes through the barrier $\}=\int_{R^{1}}|A(\lambda)|^{2} \mid\left(\left.f(\lambda)\right|^{2} d \lambda\right.$,

Prob $\{$ particle is reflected by the barrier $\}=\int_{R^{1}}|B(\lambda)|^{2} \mid\left(\left.f(\lambda)\right|^{2} d \lambda\right.$,

where $f(\lambda), \int|f(\lambda)|^{2} d \lambda=1$, is an arbitrary function; see [LL]. Since $\operatorname{Prob}\{\cdot\} \leq 1$, we necessarily obtain $|A(\lambda)|=\left|e^{i p_{\infty}(\lambda)}\right| \leq 1$ for all $\lambda$, and therefore $\Im p_{\infty}(\lambda) \geq 0$. That is a third proof that $p_{\infty}(\lambda)$ has a positive imaginary part.

\section{ACTION of $H$ 's Flows on $b(\lambda)$ AND THE ANGLES}

Now we return to the main topic. In the next two sections we demonstrate that $I(\lambda) \equiv \Im p_{\infty}(\lambda)$ produces on an invariant manifold a torus action with continuum many generators. Note that the action-angle variables for the NLS equation in the scattering case were constructed in $[\mathrm{ZM}]$. We prefer here a method of computation which uses the $\mathrm{CM}$ and is therefore different from the conventional one.

By direct computation, we obtain

$$
b^{\bullet}(\lambda)=\left\{H_{1}, b(\lambda)\right\}=-\frac{i}{2} b(\lambda) .
$$

Another useful fact is

$$
K \nabla b(\lambda)=\lambda J \nabla b(\lambda) .
$$


Then, the inductive rule

$$
\begin{aligned}
\left\{H_{n+1}, b\right\} & =i \int \nabla H_{n+1} J \nabla b(\lambda) \\
& =-i \int \nabla b(\lambda) J \nabla H_{n+1} \\
& =-i \int \nabla b(\lambda) K \nabla H_{n} \\
& =i \int \nabla H_{n} K \nabla b(\lambda) \\
& =i \lambda \int \nabla H_{n} J \nabla b(\lambda) \\
& =\lambda\left\{H_{n}, b\right\}
\end{aligned}
$$

implies

$$
b^{\bullet}(\lambda)=\left\{H_{n+1}, b\right\}=-i / 2 \lambda^{n} b(\lambda) .
$$

This means that $\theta(\lambda) \equiv-$ phase $b(\lambda)$ changes with a uniform speed; it is an angle on the invariant torus.

\section{EFfeCtive SOlution OF THE MOMENT PROBlem}

To show that $I(\lambda)$ is canonically conjugate to $\theta(\lambda)$, we establish a formula for an effective solution of the moment problem. This was introduced in $[\mathrm{V}]$ under some restrictive assumptions. Here we relax the assumptions and present an easy proof, which can be generalized to the continuum case. ${ }^{7}$

THE DISCRETE CASE. Let $H_{n}$ be a sequence of moments of some positive measure $\mu_{k}$ on $Z^{1}$ :

$$
H_{n+1}=\sum_{k} k^{n} \mu_{k}
$$

If the series

$$
s_{k}=\sum_{n=0}^{\infty} \frac{H_{n+1}}{n !}\left[\frac{\sin \pi(x-k)}{\pi(x-k)}\right]_{\left.\right|_{x=0}}^{(n)}
$$

converges absolutely for all $k$, then the measure is unique and $\mu_{k}=s_{k}$ for all $k$.

It is easy to prove the formula for compactly supported $\mu$. Take the function

$$
\frac{\sin \pi(x-k)}{\pi(x-k)}
$$

which vanishes everywhere on $Z^{1}$ except $x=k$, and consider its Taylor expansion

$$
\frac{\sin \pi(x-k)}{\pi(x-k)}=\sum_{n=0}^{\infty} \frac{x}{n !}\left[\frac{\sin \pi(x-k)}{\pi(x-k)}\right]_{\mid x=0}^{(n)} .
$$

Integration of both sides with respect to $\mu$ produces the formula. To prove it under the stated assumptions one has to consider the even and odd parts of the measure separately: $e_{n}=\left(\mu_{n}+\mu_{-n}\right) / 2, \quad o_{n}=\left(\mu_{n}-\mu_{-n}\right) / 2$. Take the even part. All moments of odd order vanish, and the even order moments match with

${ }^{7}$ For another approach to effective solution of the moment problem on the finite interval, see [F, chapter 9]. 
the corresponding moments of the original measure. The formula is true for the measure with a cut-off, which now can be removed by the bounded convergence theorem. The odd part can be considered in a similar way. The final result is produced by taking the sum of both parts. The proof is finished.

Denote by $\mathfrak{G}^{\sigma}$ the Gaussian density

$$
\mathfrak{G}^{\sigma}=\frac{1}{\sqrt{2 \pi \sigma^{2}}} e^{-\frac{x^{2}}{2 \sigma^{2}}}, \quad \sigma>0,
$$

and by $f^{\sigma}=f \otimes \mathfrak{G}^{\sigma}$ the convolution of a function or measure $f$ with the kernel $\mathfrak{G}^{\sigma}$.

The CONTINUUm CASE. Let $H_{n}$ be a sequence of moments of some measure on $R^{1}$. If the series

$$
s^{\sigma}(x)=\sum_{n=0}^{\infty} \frac{H_{n+1}}{n !}\left[\mathfrak{G}^{\sigma}(x-y)\right]_{\left.\right|_{y=0} ^{(n)}}^{(n)}
$$

converges absolutely for all $x$ and some $\sigma>0$, then the measure is unique and $s^{\sigma}(x)=\mu^{\sigma}(x)$ for all $x$.

The proof is identical to the discrete case, only now one has to integrate the Taylor expansion of $\mathfrak{G}^{\sigma}(x-y)$.

Introducing $I^{\sigma}(\lambda)$ and using the preceding formula, we get

$$
\begin{aligned}
\left\{2 I^{\sigma}\left(\lambda^{\prime}\right), \theta\left(\lambda^{\prime \prime}\right)\right\} & =\sum_{n=0}^{\infty} \frac{1}{n !}\left[\mathfrak{G}^{\sigma}\left(\lambda^{\prime}-\lambda\right)\right]_{\left.\right|_{\lambda=0} ^{(n)}}^{(n)}\left\{2 H_{n+1}, \theta\left(\lambda^{\prime \prime}\right)\right\} \\
& =\sum_{n=0}^{\infty} \frac{\left(\lambda^{\prime \prime}\right)^{n}}{n !}\left[\mathfrak{G}^{\sigma}\left(\lambda^{\prime}-\lambda\right)\right]_{\left.\right|_{\lambda=0}}^{(n)}=\mathfrak{G}^{\sigma}\left(\lambda^{\prime}-\lambda^{\prime \prime}\right) .
\end{aligned}
$$

Passing to the limit as $\sigma \downarrow 0$, we obtain

$$
\left\{I\left(\lambda^{\prime}\right), \theta\left(\lambda^{\prime \prime}\right)\right\}=\delta\left(\lambda^{\prime}-\lambda^{\prime \prime}\right)
$$

The involutive character of the $I$ 's can be proved in a similar way.

\section{The Convexity theorem}

The Convexity Theorem. For a potential from the Schwartz class $S\left(R^{1}\right)$ of complex rapidly decreasing infinitely smooth functions, the image of the momentum map is the convex cone of all nonnegative functions from the real part of $S\left(R^{1}\right)$.

The part that the map is into was already explained in section 4 . The onto part is more difficult. Assume that $b(\lambda)$ is from $S\left(R^{1}\right)$ and pure real, for definiteness. Then the theorem is proved using standard arguments, by reconstructing the potential from the scattering matrix; see $[\mathrm{M}]$. This involves a solution of the Gel'fand-Levitan integral equations for the kernels $\Gamma_{ \pm}$of the transformation operators:

$$
\Gamma_{+}(x, t)+\Omega(x+t)+\int_{x}^{\infty} \Gamma_{+}(x, s) \Omega(s+t) d s=0,
$$

where

$$
\Omega(x)=\left(\begin{array}{cc}
0 & \bar{\omega}(x) \\
\omega(x) & 0
\end{array}\right), \quad \omega(x)=\frac{1}{2 \pi} \int_{-\infty}^{+\infty} r(\lambda) e^{i \lambda x} d \lambda, \quad r(\lambda)=\frac{b(\lambda)}{a(\lambda)}
$$


and

$$
\Gamma_{-}(x, t)+\tilde{\Omega}(x+t)+\int_{-\infty}^{x} \Gamma_{-}(x, s) \tilde{\Omega}(s+t) d s=0
$$

where

$$
\tilde{\Omega}(x)=\left(\begin{array}{cc}
0 & \overline{\tilde{\omega}}(x) \\
\tilde{\omega}(x) & 0
\end{array}\right), \quad \tilde{\omega}(x)=\frac{1}{2 \pi} \int_{-\infty}^{+\infty} \tilde{r}(\lambda) e^{-i \lambda x} d \lambda, \quad \tilde{r}(\lambda)=-\frac{\bar{b}(\lambda)}{a(\lambda)} .
$$

The infinite smoothness and decay of the kernels $\Gamma_{ \pm}$are provided by the fact that $\Omega, \tilde{\Omega}$ are from $S\left(R^{1}\right)$.

The theorem implies that the conditions in section 4 which describe the function $p_{\infty}(\lambda)$ for potentials from the class $S\left(R^{1}\right)$ are not only necessary, but also sufficient for $p_{\infty}(\lambda)$ to be produced by a potential from $S\left(R^{1}\right)$.

We conjecture, by analogy with the Fourier transform (see section 7 ), that for a potential $\psi$ from the Sobolev class $H^{s}, s \geq 0$, i.e., $(1-\Delta)^{-s / 2} \psi \in L^{2}\left(R^{1}\right)$, the image of the momentum map fills up the convex cone of nonnegative functions of the weighted space $L^{1}\left(R^{1},\left(1+\lambda^{2}\right)^{s} d \lambda\right)$.

\section{Solution of the Problem}

The only condition needed on a finite number of $H_{1}, \cdots, H_{N}$ to make them correspond to some invariant manifold is

$$
\sum_{0}^{\left[\frac{N-1}{2}\right]} H_{i+j+1} \xi_{i} \xi_{j}>0, \quad \text { for any } \sum_{0}^{\left[\frac{N-1}{2}\right]} \xi_{i}^{2}>0 .
$$

In fact, this is the condition for a truncated moment problem to be definite; see $[\mathrm{A}]$.

A description of the invariant manifolds in $S\left(R^{1}\right)$ with given values of $H_{1}, \cdots, H_{N}$ reduces to a construction of the function $p_{\infty}(\lambda)$ which satisfies the conditions $i$.-iii of section 4 and has the prescribed asymptotic expansion

$$
p_{\infty}(\lambda)=-\frac{H_{1}}{\lambda}-\frac{H_{2}}{\lambda^{2}}-\cdots-\frac{H_{N}}{\lambda^{N}}-o\left(\frac{1}{\lambda^{N}}\right)
$$

for $\delta \leq \arg \lambda \leq \pi-\delta$,

We will give a description of all such functions with a given value of just one integral $H_{1}$ :

$$
p_{\infty}(\lambda)=-\frac{H_{1}}{\lambda+\varphi(\lambda)}
$$

where the arbitrary function $\varphi(\lambda)$ is such that

i. $\varphi(\lambda)=o(1)$ for $|\lambda| \rightarrow \infty$ within the angle $\delta \leq \arg \lambda \leq \pi-\delta$;

ii. $\Im \varphi(\lambda)>0$ for $\Im \lambda>0$;

iii. $\Im \varphi(\lambda) /|\lambda+\varphi(\lambda)|^{2}$ is of the class $S\left(R^{1}\right)$ on the real line.

Indeed, according to [A, section 3.4], any function $p_{\infty}(\lambda)$ with $\Im p_{\infty}(\lambda)>0$ for $\Im \lambda$ $>0$ and with the asymptotic behavior

$$
p_{\infty}(\lambda)=-\frac{H_{1}}{\lambda}-o\left(\frac{1}{\lambda}\right)
$$


within the angle $\delta \leq \arg \lambda \leq \pi-\delta$ can be represented in such a form with an arbitrary $\varphi(\lambda)$ satisfying $i$.-ii., and vice versa. The proof is easy. Consider

$$
\varphi(\lambda)=-\lambda-\frac{H_{1}}{p_{\infty}(\lambda)}, \quad \text { with } p_{\infty}(\lambda)=\frac{1}{\pi} \int \frac{d \mu_{\infty}(t)}{t-\lambda}, \quad \int d \mu_{\infty}(t)=\pi H_{1} .
$$

This implies the condition $i$. Now

$$
\Im \varphi(\lambda)=-\Im \lambda+\Im \lambda \int \frac{d \mu_{\infty}}{|t-\lambda|^{2}} \frac{\pi H_{1}}{\left|\int \frac{d \mu_{\infty}}{t-\lambda}\right|^{2}} .
$$

By the Schwartz inequality,

$$
\left|\int \frac{d \mu_{\infty}}{t-\lambda}\right|^{2} \leq \int d \mu_{\infty} \int \frac{d \mu_{\infty}}{|t-\lambda|^{2}}=\pi H_{1} \int \frac{d \mu_{\infty}}{|t-\lambda|^{2}}
$$

and this implies the condition ii. The proof of converse statement is trivial. In order to get $p_{\infty}(\lambda)$ which corresponds to some invariant manifold in $S\left(R^{1}\right)$ we need to impose some restrictions on $\varphi(\lambda)$. For real $\lambda$,

$$
\Im p_{\infty}(\lambda)=\frac{H_{1} \Im \varphi(\lambda)}{|\lambda+\varphi(\lambda)|^{2}}
$$

This produces the additional condition iii. Obviously, there are plenty of suitable functions $\varphi$.

The case of general $N<\infty$ can be considered in a similar way with the aid of the so-called Schur algorithm; see [A, section 3.3].

\section{REFERENCES}

[AKNS] M.J. Ablowitz, D.J. Kaup, A.C. Newell A.C. and H. Segur, The inverse scattering transform-Fourier analysis for nonlinear problems, Stud. Appl. Math. 53 (1974), 249315. MR 56:9018

[A] N.I. Akhiezer, The Classical Moment Problem and Some Related Questions of Analysis, Oliver and Boyd, Edinburgh, 1965. MR 32:1518

[AT] M. Atiyah, Convexity and commuting Hamiltonians., Bull. London Math. Soc. 14 (1982), 1-15. MR 83e:53037

[BDZ] R. Beals, P. Deift and X. Zhou, The inverse scattering transform on the line, Important Developments in Soliton Theory (V.E. Zakharov, ed.), Springer-Verlag, Berlin, 1993, pp. 7-32. MR 95k:34020

[B] F. Bloch, Über die Quantenmechanik der Elektronen in Kristallgittern, Z. Phys 52 (1929), 555-600.

[CL] E. Coddington and N. Levinson, Theory of Ordinary Differential Equation, McGraw-Hill, New York, 1955. MR 16:1022b

[FT] L.D. Faddeev and L.A. Takhtajan, Hamiltonian Methods in the Theory of Solitons, Springer-Verlag, Berlin, 1987. MR 89m:58103

[F] W. Feller, Introduction to Probability Theory and its Applications, Vol. II, 2nd ed., Wiley, New York, 1971. MR 42:5292

[GS] V. Guillemin and S. Sternberg, Convexity property of the momentum map, Invent. Math. 67 (1982), 491-513. MR 83m:58037

[KO] P. Koosis, Introduction to $H^{p}$ spaces, Cambridge Univ. Press, Cambridge, 1980. MR 81c:30062

[K] M.G. Krein, On the theory of accelerant and S-matrices of canonical differential systems, Dokl Akad Nauk SSSR 111 (1956), 1167-1170. (Russian) MR 19:277f

[KMA] M.G. Krein and P.E. Melik-Adamian, A contribution to the theory of S-matrices of canonical differential equations with summable potential, Dokl. Akad Nauk Armjan. SSR 46 (1968), 150-155; English transl., M. G. Krein, Topics in differential and integral equations and operator theory, Birkhäuser, Basel, 1983, pp. 295-301. MR 53:13715; MR 86m:00014 
[LL] L.D. Landau and E.M. Lifshitz, Quantum Mechanics, Course in Theoretical Physics, vol. 3, Pergamon Press, Oxford, 1958.

[LS] B.M. Levitan and I. S. Sargsian, Sturm-Liouwille and Dirac Operators, Kluwer Academic, Dordrecht, 1991. MR 92i:34119

[M] A.M. Marchenko, Sturm-Liouwille Operators and Their Applications, Birkhauser, Basel, 1986. MR 88f:34034

[MO] V.A. Marchenko and I.V. Ostrovskii, A characterization of the spectrum of Hill's operator, Math. USSR-Sbornik 97 (1975), 493-554. MR 53:13717

[MC1] H.P. McKean, Compatible Brackets in Hamiltonian Mechanics, Important Developments in Soliton Theory (V.E. Zakharov and A.S. Fokas, eds.), Springer-Verlag, Berlin, Heidelberg New York, 1993, pp. 344-354. MR 95c:58097

[MC2] H.P. McKean, Geometry of KdV (1): Additive and the Unimodular Spectral Classes, Revista Matematica Iberoamericana 2 (1987), 235-261. MR 89b:58096

[MCV] H.P. McKean and K.L. Vaninsky, Action-angle variables for nonlinear Schrödinger equation, preprint, 1995.

[MA] P.E. Melik-Adamian, On the properties of S-matrices of canonical differential equations on the entire line, Akad. Nauk Armjan. SSR Dokl 58 (1974), 199-205. MR 50:7719

[V] K.L. Vaninsky, Invariant Gibbsian measures of the Klein-Gordon equation, Stochastic Analysis (M.C. Cranston and M.A. Pinsky, eds.), Proc. Sympos. Pure Math., vol. 57, American Mathematical Society, 1995, pp. 495-510. MR 96g:58115

[ZS] V.E. Zakharov and A.B. Shabat, Exact theory of two-dimensional self-focusing and onedimensional self-modulation of waves in nonlinear media, Soviet Phys. JETP 34 (1972), 62-69. MR 53:9966

[ZM] V.E.Zakharov and S.V. Manakov, On the complete integrability of the nonlinear Schrödinger equation, Theor. Math. Phys. 19 (1975), 551-560. MR 57:8636

Department of Mathematics, Kansas State University, Manhattan, Kansas 66506

E-mail address: vaninsky@math.ksu.edu 